\title{
Comparison between cap-assisted and ligation-assisted endoscopic mucosal resection for rectal neuroendocrine tumors
}

\author{
Jin Lee ${ }^{a}$, Yong Eun Parka, Joon Hyuk Choia, Nae-Yun Heo a, Jongha Parka, Seung Ha Parka, Young Soo \\ Moon ${ }^{a}$, Kyung Han Namb, Tae Oh Kima
}

Inje University Haeundae Paik Hospital, Busan, Republic of Korea

\section{Abstract}

\begin{abstract}
Background Modified endoscopic mucosal resection (EMR) is considered a treatment option for rectal neuroendocrine tumors (NETs) $<10 \mathrm{~mm}$ in diameter. In this study, we evaluated the clinical outcomes of cap-assisted EMR (EMR-C) and EMR with a ligating device (EMR-L).
\end{abstract}

Methods We retrospectively analyzed 158 patients with 162 rectal NETs treated endoscopically at a single Korean tertiary hospital between March 2010 and November 2017. We evaluated the rates of endoscopic en bloc resection, histologic complete resection, and procedural complications according to the treatment method.

Results Among 162 rectal NETs, 42 were treated with EMR-C and 120 with EMR-L. The endoscopic en bloc resection rate was higher in the EMR-L group than in the EMR-C group (100\% vs. $92.9 \%, \mathrm{P}=0.003$ ). A trend was observed towards a superior histologic complete resection rate in the EMR-L group, but it was not statistically significant $(92.5 \%$ vs. $83.3 \%, \mathrm{P}=0.087)$. There were no significant differences in procedural complications $(\mathrm{P}=0.870)$. In a multivariate analysis, a tumor located $\geq 10 \mathrm{~cm}$ from the anal verge was related to histologic incomplete resection $(\mathrm{P}=0.039)$.

Conclusion EMR-L may be the preferable treatment method, considering both endoscopic en bloc resection rate and histologic complete resection rate.

Keywords Rectal neuroendocrine tumor, endoscopic mucosal resection-ligating device (EMR-L), cap-assisted EMR (EMR-C)

Ann Gastroenterol 2020; 33 (4): 1-6

\section{Introduction}

Rectal neuroendocrine tumors (NETs) account for roughly one third of all gastrointestinal NETs, with the rectum being the second most common site (34.6\%) after the small intestine (36.5\%) [1]. Recently, incidences of rectal NETs have been increasing. This may be due to an increase in screening colonoscopies, improvement in detection rates by advanced

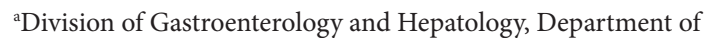
Internal Medicine (Jin Lee, Yong Eun Park, Joon Hyuk Choi, Nae-Yun Heo, Jongha Park, Seung Ha Park, Young Soo Moon, Tae Oh Kim); 'Department of Pathology (Kyung Han Nam), Haeundae Paik Hospital, College of Medicine, Inje University, Republic of Korea

\section{Conflict of Interest: None}

Correspondence to: Tae Oh Kim, Division of Gastroenterology, Department of Internal Medicine, Haeundae Paik Hospital, College of Medicine, Inje University, 1435 Jwa-dong, Haeundae-gu, Busan 612896, Republic of Korea, e-mail: kto0440@paik.ac.kr

Received 8 October 2019; accepted 5 February 2020; published online 10 May 2020

DOI: https://doi.org/10.20524/aog.2020.0485 endoscopic devices, and increased clinical awareness by endoscopists [1-4]. The majority of NETs are asymptomatic and are detected accidentally at an early stage. Given the low risk of metastasis [5,6] and their benign behavior [7], it is recommended that small rectal NETs less than $10 \mathrm{~mm}$ in size can be treated by endoscopic resection if there is no lymphovascular invasion, muscularis invasion, or lymph node metastasis [8]. Several studies also suggest that selected tumors $10-15 \mathrm{~mm}$ in size can be removed endoscopically if there is no lymph node metastasis or muscularis invasion [5,9-11].

Various endoscopic treatments, such as conventional endoscopic mucosal resection (EMR), modified EMR, and endoscopic submucosal dissection (ESD), are used to remove rectal NETs. Several studies report that complete resection rates of conventional EMR are variable, ranging from 3070\% [12-16]. Modified EMR includes cap-assisted EMR (EMR-C), EMR with a ligating device (EMR-L), and EMR after circumferential precutting (EMR-P). Recent metaanalyses showed that modified EMR and ESD are preferable treatment methods to conventional EMR for the endoscopic resection of rectal NETs, because of their high rates of complete tumor resection $[17,18]$. The ESD method requires extensive endoscopic experience and the procedure takes more time. 
Modified EMR is widely used because of its short procedure time and low complication rate compared to ESD. Among the modified EMR techniques, EMR-C and EMR-L are commonly performed, with EMR-P being used more recently.

It is not well established which method of modified EMR provides a better outcome. In general, the choice is typically determined by the operator's preference, along with any cost and equipment considerations. Therefore, the aim of this study was to compare the clinical outcomes of EMR-C and EMR-L. We also evaluated the factors associated with histologic incomplete resection.

\section{Patients and methods}

\section{Patients}

We performed a retrospective medical chart review and collected data on patients with rectal NETs treated endoscopically at a single Korean tertiary hospital from March 2010 to November 2017. The characteristics of the patients and rectal NETs, as well as the clinical outcomes of endoscopic resection, were analyzed. Abdominal and pelvic computed tomography (CT) and chest radiography were performed in all patients to assess metastasis. Out of 177 patients, 19 were treated using conventional EMR, 40 using EMR-C, and 118 using EMR-L. After excluding the 19 patients who underwent conventional EMR, 158 patients with 162 rectal NETs were enrolled in this study. This study was approved by the Institutional Review Board of our hospital. Informed consent was not obtained because this was a retrospective study.

\section{Endoscopic treatment methods}

All endoscopic treatments were conducted by 3 highly experienced endoscopists, who have each performed over 6000 colonoscopies. We recommended EMR-C and EMR-L for rectal NETs $<10 \mathrm{~mm}$ in diameter and surgery for NETs $\geq 20$ $\mathrm{mm}$ in diameter. For NETs of $\geq 10 \mathrm{~mm}$ in diameter, endoscopic ultrasonography was recommended to assess the exact size and the depth of invasion before treatment. Endoscopic resection was considered when the tumor was confined to submucosa and was less than $15 \mathrm{~mm}$ in size. For NETs sized 16-20 mm, either endoscopic submucosal dissection or surgery was recommended, taking into account the patient's preference and the risk of metastasis. The resection methods for rectal NETs were determined according to the endoscopist's preference. All procedures were performed in the outpatient endoscopy clinic.

The EMR-C procedure was carried out as follows (Fig. 1). A single-channel endoscope (GIF-H260, Olympus, Japan) was used with a transparent cap (reusable oblique distal attachments with rim, MAJ-290, Olympus, Japan) and a crescent-type snare (SD-221L-25, Olympus, Japan). First, a 0.9\% saline solution mixed with epinephrine and indigo carmine was injected beneath the tumor to lift the lesion. After a transparent cap had been fitted to the scope, the crescent-type snare was looped along the inner ridge of the cap. Endoscopic suctioning of the tumor into the cap was performed, followed by closure of the snare.

For the EMR-L technique (Fig. 2), a $0.9 \%$ saline solution mixed with epinephrine and indigo carmine was injected into the submucosal layer of the tumor to elevate the lesion. The endoscopic scope was equipped with a ligating device (Stigmann-Goff Clearvu Endoscopic ligator, Conmed, Mexico). After aspiration into the ligating device and deployment of the elastic band, snare resection was performed below the band.
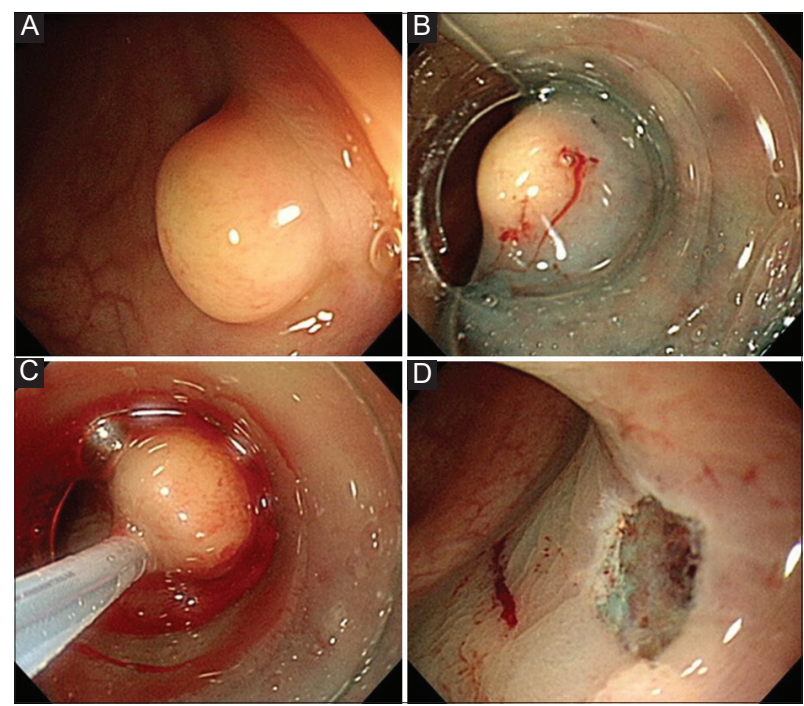

Figure 1 Cap-assisted endoscopic mucosal resection. (A) A yellowish tumor was detected; (B) adequate lift after submucosal injection; (C) endoscopic suctioning of the tumor into the cap followed by closure of the snare; (D) complete resection with no signs of perforation or residual tissue
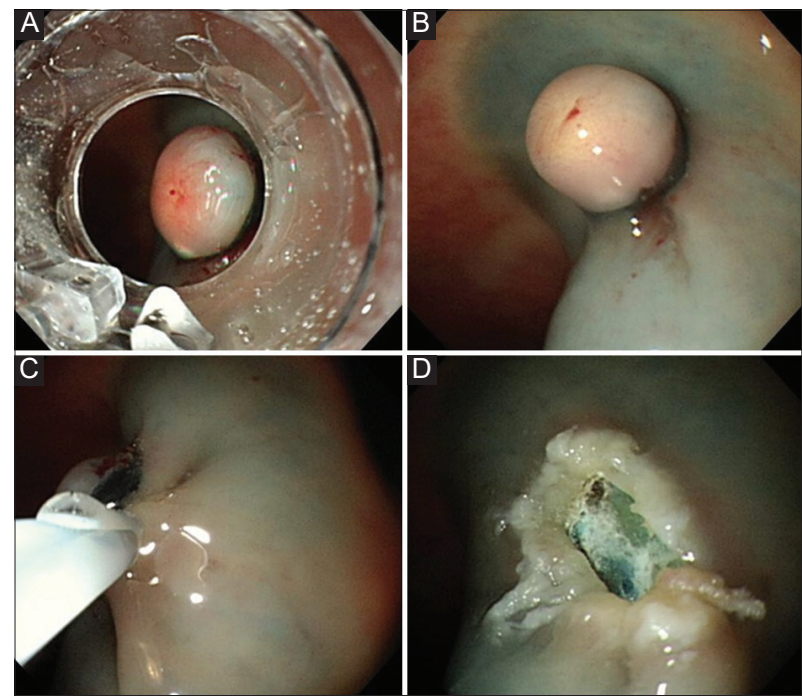

Figure 2 Endoscopic mucosal resection with a ligating device. (A) Aspiration of a tumor into the ligating device; (B) deployed elastic band; (C) snare resection performed below the band; (D) complete resection with no signs of perforation or residual tissue 


\section{Outcomes analysis}

The histopathologic findings, such as tumor size, depth of invasion, presence of lymphovascular invasion, and status of deep and lateral resection margins, were evaluated by an experienced pathologist. Immunohistochemical staining for neuron-specific enolase and synaptophysin was done to support the diagnosis. Each specimen was graded based on mitotic count and Ki-67 index, according to the World Health Organization's classification.

Endoscopic en bloc resection was defined as a resection in one piece during a procedure, and histologic complete resection (H-CR) was defined as a status of no involvement of the tumor in vertical and lateral margins. The procedure time was defined as the period from submucosal injection of the saline mixture to endoscopic resection of the tumor.

\section{Statistical analysis}

Data were expressed as mean \pm standard deviations (SD) for continuous variables and as frequencies and percentages for categorical variables. Differences in patient and tumor characteristics were compared using independent $t$-tests for numerical variables and the chi-square test for categorical variables. Factors related to incomplete histologic resection were assessed using logistic regression analysis. All statistical analyses were performed using MedCalc Statistical Software v.16.2.1 (MedCalc Software, Mariakerke, Belgium). A P-value $<0.05$ was considered to be statistically significant.

\section{Results}

\section{Baseline characteristics}

A total of 158 patients with 162 rectal NETs were treated endoscopically. Among 162 rectal NETs, 42 lesions were resected by the EMR-C method and 120 lesions using EMR-L. Four patients had multiple synchronous rectal NETs, 2 NETs in each case. The mean age of enrolled patients was 51.0 (range: 27-80) years, with 91 male (57.6\%) and 67 female patients (42.4\%). The mean size of tumors was $4.8 \pm 2.0$ (range: $1.0-12.0$ ) $\mathrm{mm}$, with mean distance from the anal verge $7.8 \pm 3.0$ (range: $1.0-15.0) \mathrm{cm}$. Before referral to our hospital, endoscopic biopsy was done in 23 (54.8\%) lesions in the EMR-C group and 51 lesions $(42.5 \%)$ in the EMR-L group. The characteristics of patients and tumors are summarized in Table 1.

\section{Clinical outcomes}

The outcomes of EMR-C and EMR-L are summarized in Table 2. Overall, the endoscopic en bloc resection rate for both groups (EMR-C and EMR-L) was $98.1 \%$ and the $\mathrm{H}-\mathrm{CR}$ rate was $90.1 \%$. The endoscopic en bloc resection rate was higher in the EMR-L group than in the EMR-C group (100\% vs. $92.9 \%$, $\mathrm{P}=0.003)$. A trend was observed towards a superior $\mathrm{H}-\mathrm{CR}$ rate in the EMR-L group, but it was not statistically significant (92.5\% vs. $83.3 \%, \mathrm{P}=0.087)$. Among a total of 16 patients with histologic incomplete resection, the vertical margin was positive in all patients. In 2 patients, both vertical and lateral margins were positive. In most cases, resected tumors were located in the center of the submucosa in the histopathologic image, so all specimens with histologic incomplete resection were positive for the vertical margin, while only 2 cases were positive for both vertical and lateral margins (Fig. 3). There was no difference in procedure time between the 2 groups. Complications occurred in 7 patients (4.4\%): 5 patients had

Table 1 Baseline characteristics of patients and tumors

\begin{tabular}{|c|c|c|c|}
\hline Characteristics & $\begin{array}{l}\text { EMR-C } \\
(\mathrm{n}=42)\end{array}$ & $\begin{array}{l}\text { EMR-L } \\
(n=120)\end{array}$ & P-value \\
\hline Age (years) & $49.2 \pm 12.2$ & $51.7 \pm 10.7$ & 0.208 \\
\hline Male sex & $23(54.8)$ & $68(56.7)$ & 0.831 \\
\hline Tumor size (mm) & $4.6 \pm 2.3$ & $4.8 \pm 1.9$ & 0.590 \\
\hline $\begin{array}{l}\text { Distance from anal verge } \\
(\mathrm{cm})\end{array}$ & $7.2 \pm 3.4$ & $7.9 \pm 2.8$ & 0.179 \\
\hline $\begin{array}{l}\text { Presence of previous } \\
\text { biopsy }\end{array}$ & $23(54.8)$ & $51(42.5)$ & 0.171 \\
\hline Grade of NETs & & & 0.078 \\
\hline Grade 1 & $39(92.9)$ & $118(98.3)$ & \\
\hline Grade 2 & $3(7.1)$ & $2(1.7)$ & \\
\hline Grade 3 & $0(0)$ & $0(0)$ & \\
\hline Lymphovascular invasion & & & 0.152 \\
\hline Yes & $1(2.4)$ & $1(0.8)$ & \\
\hline No & $6(14.3)$ & $31(25.8)$ & \\
\hline Not identified & $35(83.3)$ & $88(73.3)$ & \\
\hline Depth of tumor & & & 0.599 \\
\hline Mucosa & $1(2.4)$ & $5(4.2)$ & \\
\hline Submucosa & $41(97.6)$ & $115(95.8)$ & \\
\hline $\begin{array}{l}\text { Muscularis propria or } \\
\text { deeper }\end{array}$ & $0(0)$ & $0(0)$ & \\
\hline
\end{tabular}

Values are mean \pm standard deviation or number (\%)

EMR-C, cap-assisted endoscopic mucosal resection; EMR-L, endoscopic mucosal resection with ligating device

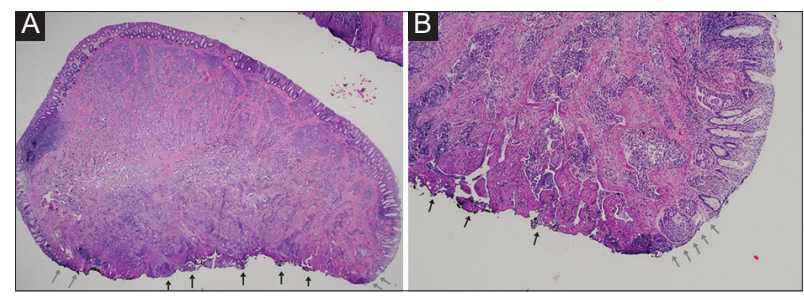

Figure 3 Histopathologic images of a rectal neuroendocrine tumor with both vertical and lateral margin involvements. Vertical (black arrow) and lateral (gray arrow) margin involvements are noted. (A) H\&E stain, $\times 1.5$; $(\mathrm{B}) \times 100$ 
Table 2 Clinical outcomes of EMR-C and EMR-L

\begin{tabular}{|c|c|c|c|c|}
\hline Outcomes & Overall $(n=162)$ & EMR-C $(n=42)$ & EMR-L $(n=120)$ & P-value \\
\hline Endoscopic complete resection & $159(98.1)$ & $39(92.9)$ & $120(100)$ & 0.003 \\
\hline Histologic complete resection & $146(90.1)$ & $35(83.3)$ & $111(92.5)$ & 0.087 \\
\hline Vertical margin positive & $14(8.6)$ & $6(14.3)$ & $8(6.7)$ & 0.131 \\
\hline Vertical and lateral margin positive & $2(1.2)$ & $1(2.4)$ & $1(0.8)$ & 0.435 \\
\hline Procedure time $(\mathrm{min})$ & $5.5 \pm 5.2$ & $5.5 \pm 2.5$ & $5.5 \pm 5.9$ & 0.983 \\
\hline Complication & $7(4.3)$ & $2(4.8)$ & $5(4.2)$ & 0.870 \\
\hline Intraprocedural bleeding & $5(3.1)$ & $1(2.4)$ & $4(3.3)$ & 0.759 \\
\hline Delayed bleeding & $1(0.6)$ & $0(0)$ & $1(0.8)$ & 0.554 \\
\hline Perforation & $1(0.6)$ & $1(2.4)$ & $0(0)$ & 0.091 \\
\hline
\end{tabular}

EMR-C, cap-assisted endoscopic mucosal resection; EMR-L, endoscopic mucosal resection with ligating device

intra-procedural bleeding, 1 had delayed bleeding, and 1 had a perforation. Complications were treated with endoscopic procedures such as clipping or coagulation therapy. There were no significant differences in the margin status between the 3 operators $(\mathrm{P}>0.05)$.

\section{Follow up and recurrence}

During a 26.2-month (range: 2.6-96.0) median follow-up period, surveillance endoscopic evaluations were performed in 105 (66.5\%) patients and abdominopelvic CT (APCT) was performed to assess metastatic recurrence in $80(50.6 \%)$ patients. Among 16 patients histologically incompletely resected, 13 underwent follow-up evaluation with APCT and colonoscopy. No evidence of recurrence was seen on CT and endoscopy.

\section{Risk factors for histologic incomplete resection of rectal NETs}

In univariate and multivariate analyses, a tumor located $\geq 10$ $\mathrm{cm}$ from the anal verge was related to histologic incomplete resection (Table 3). Odds ratios were 2.9 (95\% confidence interval $[\mathrm{CI}] 1.00-8.49 ; \mathrm{P}=0.048)$ in univariate analysis and $3.1(95 \%$ CI $1.05-9.26 ; \mathrm{P}=0.039)$ in multivariate analysis. The tumor size, presence of previous biopsy, and method of EMR were not associated with incomplete histologic resection in multivariate analysis.

\section{Discussion}

Various endoscopic treatments, such as conventional EMR, modified EMR and ESD, are used to remove rectal NETs. Modified EMR is widely used because of its high complete resection rates, short procedure times and low complication rates. Among modified EMR methods, EMR-C and EMR-L are commonly used, with EMR-P also used more recently.
EMR-L is the one of the most commonly used methods for modified EMR. EMR-L differs from EMR as the lesion is suctioned into the ligating device and an elastic band is deployed before snare resection. In a study by Mashimo et al [19], H-CR using EMR-L was $95.2 \%(n=63)$, similar to the rate reported for ESD. In another relatively large-scale study comparing EMR-L $(n=45)$ and EMR $(n=55)$, the overall H-CR was significantly higher for EMR-L than for EMR (93.3\% vs. $65.5 \%)$ [16]. Other studies showed similar results, such as an H-CR of EMR-L at $93.9 \%(n=33)$ and $100 \%(n=40)[20,21]$. An analysis of 17 studies reported that the complete resection rate of rectal NETs using EMR-L was $94.8 \%(n=248)$ compared with $72.4 \%$ for EMR-C $(n=152)$ [5]. Most previous studies have shown a high rate of complete resection in their EMR-L groups.

EMR-C is a widely used treatment method for rectal NETs. Previous studies [13,22-25] reported that EMR-C is an effective method for the endoscopic resection of rectal NETs. However, its $\mathrm{H}-\mathrm{CR}$ rate varies from $71.7-100 \%$, while the studies included relatively small sample sizes. Some studies have also reported low rates of complete resection [22,23], while others have shown relatively high rates $[24,25]$. Kim et al found a relatively low $\mathrm{H}-\mathrm{CR}$, at $83.3 \%$, but their sample size was very small $(\mathrm{n}=6)$ [22]. Son et al [23] also reported tumor-free margins in $71.7 \%$ of 53 patients treated with EMR-C. However, Zhao et al $(\mathrm{n}=10)$ reported that the H-CR of EMR-C was $100 \%$ [24], while Park et al $(\mathrm{n}=65)$ compared the outcomes of EMR-C with those of ESD and reported high rates of endoscopic en bloc resection and $\mathrm{H}-\mathrm{CR}$ (100\% and $92.3 \%$, respectively) in the EMR-C group [25]. Yang et al $(\mathrm{n}=34)$ found similar results, with an $\mathrm{H}-\mathrm{CR}$ of $94.1 \%$ [13]. As mentioned above, the complete resection rate of EMR-C was not consistently high compared with that of EMR-L. These different results among studies, which each assessed the clinical outcomes of EMR-C in a single hospital, may be attributable to the relatively small sample sizes; hence, further large-scale studies are needed to clarify this issue.

According to many previous studies, EMR-L is thought to be superior, or similar, to EMR-C in terms of complete resection rates of rectal NETs. In this study, we evaluated the clinical outcomes of EMR-C and EMR-L and found that the 
Table 3 Risk factors for positive resection margin of rectal neuroendocrine tumor

\begin{tabular}{|c|c|c|c|c|}
\hline \multirow[t]{2}{*}{ Risk factors } & \multicolumn{2}{|c|}{ Univariate analysis } & \multicolumn{2}{|c|}{ Multivariate analysis } \\
\hline & OR $(95 \% \mathrm{CI})$ & P-value & OR $(95 \% \mathrm{CI})$ & P-value \\
\hline Tumor size $\geq 5 \mathrm{~mm}$ (vs. $<5 \mathrm{~mm})$ & $1.3(0.49-3.94)$ & 0.529 & $1.4(0.47-4.15)$ & 0.532 \\
\hline EMR-C (vs. EMR-L) & $2.4(0.85-7.10)$ & 0.094 & $2.6(0.87-7.80)$ & 0.086 \\
\hline Presence of previous biopsy & $0.9(0.32-2.59)$ & 0.870 & $0.7(0.26-2.37)$ & 0.679 \\
\hline Distance from anal verge $(\geq 10 \mathrm{~cm})$ & $2.9(1.00-8.49)$ & 0.048 & $3.1(1.05-9.26)$ & 0.039 \\
\hline
\end{tabular}

EMR-C, cap-assisted endoscopic mucosal resection; EMR-L, endoscopic mucosal resection with ligating device;

$\mathrm{OR}$, odds ratio; $\mathrm{CI}$, confidence interval

$\mathrm{H}-\mathrm{CR}$ rate did not differ significantly between the 2 groups (92.5\% vs. $83.3 \%, \mathrm{P}=0.087$ ). However, a numerical trend was observed towards a superior H-CR rate in the EMR-L group, while the endoscopic en bloc resection rate was significantly higher in the EMR-L group than in the EMR-C group (100\% vs. $92.9 \%, \mathrm{P}=0.003)$. These results could be due to several reasons. For example, since the band thickness used in EMR-L is larger than the thickness of the snare in EMR-C, lateral and deep margins are more easily secured in EMR-L. Additionally, EMR-L uses a technique that resects the tumor by snaring below the band ligation. Although procedural time did not differ significantly between the 2 treatments, until sufficient skill had been acquired through experience it was slightly more difficult to position a snare along the inner ridge of the cap in EMR-C, rather than simply ligating a tumor as in EMR-L. The economic factor may be another factor to consider; that is, the cost-benefit should be considered in the selection of endoscopic treatment method. In the case of EMR-L, the cost of the ligating device is about 20,000 KRW (17 USD). In EMR-C the oblique cap can be reused, making the cost the same as that of conventional EMR. There may be differences in the coverage of cost in medical care, depending on the insurance system in each country, which might influence the selection of treatment methods from the socioeconomic point of view.

Several previous studies [25-28] have reported factors relating to incomplete resection of rectal NETs. However, there are conflicting data regarding which factors, including tumor size and tumor location, are predictors of incomplete resection. Park et al [25] demonstrated that treatment method was the only significant factor related to incomplete resection. Lee et al [26] showed that both male sex and the treatment methods used were significantly associated with incomplete resection. Kim et al [27] found that H-CR was related to the tumor size, regardless of treatment method. Conflicting findings have also been reported regarding the association between tumor location and incomplete resection. The H-CR for lesions was significantly higher in the lower rectum than in the upper rectum (98.3\% vs. 50\%) [19]. However, another study reported that the location of tumors was not significantly associated with complete resection rates when EMR-L was conducted, in contrast to the results of other EMR methods [16]. In this study, the H-CR was higher in the tumors located $<10 \mathrm{~cm}$ from the anal verge than in those located $\geq 10 \mathrm{~cm}$ from the anal verge
(93.9\% vs. $84.1 \%$ ), consistent with the results of a previous study [19].

This study had several limitations which need to be addressed. First, it was performed at a single tertiary hospital; thus, the sample size was relatively small and may be insufficient for the generalization of results. Second, there was no randomization of treatment group, as this study was retrospective. Therefore, the number of patients in the EMR-L group was relatively high compared with that in the EMR-C group. Third, the follow-up period was relatively short, and therefore insufficient to assess the recurrence or prognosis of rectal NETs.

In conclusion, although both EMR-C and EMR-L are acceptable treatment methods in the endoscopic resection of rectal NETs, EMR-L may be the preferable method, considering both endoscopic en bloc resection rate and histologic complete resection rate. It would be better to select an appropriate treatment method taking the endoscopist's preference into account.

\section{Summary Box}

\section{What is already known:}

- Modified endoscopic mucosal resection (EMR) is considered a treatment option for rectal neuroendocrine tumors (NETs)

- Recent studies showed that modified EMR and endoscopic submucosal dissection are preferable to conventional EMR for the endoscopic resection of rectal NETs

- It has not been well established which method of modified EMR provides a better outcome

\section{What the new findings are:}

- Both EMR-C and EMR-L are acceptable treatments in the endoscopic resection of rectal NETs

- EMR-L may be the preferable treatment method, considering both endoscopic en bloc resection rate and histologic complete resection rate 


\section{References}

1. Tsikitis VL, Wertheim BC, Guerrero MA. Trends of incidence and survival of gastrointestinal neuroendocrine tumors in the United States: a SEER analysis. J Cancer 2012;3:292-302.

2. Hauso O, Gustafsson BI, Kidd M, et al. Neuroendocrine tumor epidemiology: contrasting Norway and North America. Cancer 2008;113:2655-2664.

3. Cho MY, Kim JM, Sohn JH, et al; Gastrointestinal Pathology Study Group of Korean Society of Pathologists. Current trends of the incidence and pathological diagnosis of gastroenteropancreatic neuroendocrine tumors (GEP-NETs) in Korea 2000-2009: multicenter study. Cancer Res Treat 2012;44:157-165.

4. Kim BC, Park CH, Kim TI, et al. Variable clinical classifications and diagnostic coding systems of colorectal neuroendocrine tumor. Intest Res 2013;11:14-22.

5. de Mestier L, Brixi H, Gincul R, Ponchon T, Cadiot G. Updating the management of patients with rectal neuroendocrine tumors. Endoscopy 2013;45:1039-1046.

6. Sun JM, Jung HC. Gastrointestinal carcinoid tumor. Korean J Gastroenterol 2004;44:59-65.

7. Kim GU, Kim KJ, Hong SM, et al. Clinical outcomes of rectal neuroendocrine tumors $\leq 10 \mathrm{~mm}$ following endoscopic resection. Endoscopy 2013;45:1018-1023.

8. Caplin M, Sundin A, Nillson O, et al; Barcelona Consensus Conference participants. ENETS consensus guidelines for the management of patients with digestive neuroendocrine neoplasms: colorectal neuroendocrine neoplasms. Neuroendocrinology 2012;95:88-97.

9. Park CH, Cheon JH, Kim JO, et al. Criteria for decision making after endoscopic resection of well-differentiated rectal carcinoids with regard to potential lymphatic spread. Endoscopy 2011;43:790-795.

10. Park HW, Byeon JS, Park YS, et al. Endoscopic submucosal dissection for treatment of rectal carcinoid tumors. Gastrointest Endosc 2010;72:143-149.

11. Kwaan MR, Goldberg JE, Bleday R. Rectal carcinoid tumors: review of results after endoscopic and surgical therapy. Arch Surg 2008;143:471-475.

12. Kim KM, Eo SJ, Shim SG, et al. Treatment outcomes according to endoscopic treatment modalities for rectal carcinoid tumors. Clin Res Hepatol Gastroenterol 2013;37:275-282.

13. Yang DH, Park Y, Park SH, et al. Cap-assisted EMR for rectal neuroendocrine tumors: comparisons with conventional EMR and endoscopic submucosal dissection (with videos). Gastrointest Endosc 2016;83:1015-1022.

14. Nakamura K, Osada M, Goto A, et al. Short- and long-term outcomes of endoscopic resection of rectal neuroendocrine tumours: analyses according to the WHO 2010 classification. Scand J Gastroenterol 2016;51:448-455.

15. Jeon JH, Cheung DY, Lee SJ, et al. Endoscopic resection yields reliable outcomes for small rectal neuroendocrine tumors. Dig Endosc 2014;26:556-563.

16. Kim HH, Park SJ, Lee SH, et al. Efficacy of endoscopic submucosal resection with a ligation device for removing small rectal carcinoid tumor compared with endoscopic mucosal resection: analysis of 100 cases. Dig Endosc 2012;24:159-163.

17. He L, Deng T, Luo H. Efficacy and safety of endoscopic resection therapies for rectal carcinoid tumors: a meta-analysis. Yonsei Med J 2015;56:72-81

18. Zhou X, Xie H, Xie L, Li J, Cao W, Fu W. Endoscopic resection therapies for rectal neuroendocrine tumors: a systematic review and meta-analysis. J Gastroenterol Hepatol 2014;29:259-268.

19. Mashimo Y, Matsuda T, Uraoka T, et al. Endoscopic submucosal resection with a ligation device is an effective and safe treatment for carcinoid tumors in the lower rectum. J Gastroenterol Hepatol 2008;23:218-221.

20. Lee SH, Park SJ, Kim HH, et al. Endoscopic resection for rectal carcinoid tumors: comparison of polypectomy and endoscopic submucosal resection with band ligation. Clin Endosc 2012;45:89-94.

21. Kim KM, Eo SJ, Shim SG, et al. Treatment outcomes according to endoscopic treatment modalities for rectal carcinoid tumors. Clin Res Hepatol Gastroenterol 2013;37:275-282.

22. Kim YJ, Lee SK, Cheon JH, et al. Efficacy of endoscopic resection for small rectal carcinoid: a retrospective study. Korean J Gastroenterol 2008;51:174-180.

23. Son HJ, Sohn DK, Hong CW, et al. Factors associated with complete local excision of small rectal carcinoid tumor. Int J Colorectal Dis 2013;28:57-61.

24. Zhao ZF, Zhang N, Ma SR, et al. A comparative study on endoscopy treatment in rectal carcinoid tumors. Surg Laparosc Endosc Percutan Tech 2012;22:260-263.

25. Park SB, Kim HW, Kang DH, Choi CW, Kim SJ, Nam HS. Advantage of endoscopic mucosal resection with a cap for rectal neuroendocrine tumors. World J Gastroenterol 2015;21:9387-9393.

26. Lee HJ, Kim SB, Shin CM, et al. A comparison of endoscopic treatments in rectal carcinoid tumors. Surg Endosc 2016;30:3491-3498.

27. Kim J, Kim JH, Lee JY, Chun J, Im JP, Kim JS. Clinical outcomes of endoscopic mucosal resection for rectal neuroendocrine tumor. BMC Gastroenterol 2018;18:77.

28. Choi CW, Park SB, Kang DH, et al. The clinical outcomes and risk factors associated with incomplete endoscopic resection of rectal carcinoid tumor. Surg Endosc 2017;31:5006-5011. 